\title{
Machine learning and artificial intelligence research for patient benefit: 20 critical questions on transparency, replicability, ethics, and effectiveness
}

\author{
(c) (i) OPEN ACCESS
}

An error was included in the accepted version of this paper by Vollmer and colleagues (BMJ 2020;368:16927, doi:10.1136/ bmj.16927, published 20 March 2020), and was not picked up before publication. In the author list, David Grainger was spelt incorrectly.

The paper will be amended in due course. 